\title{
Mucopolisacaridosis, la anestesia regional es nuestra mejor aliada
}

\author{
Vizgarra GN. ${ }^{1}$ \\ 1 Hospital Regional Dr. Ramón Carrillo, Santiago del Estero, Argentina.
}

Introducción: Las MPS son un grupo de enfermedades genéticas en donde la deficiencia de las enzimas que metabolizan los glicosoaminoglicanos hace que haya un deposito anormal de ellos en todo tipo de tejido. Con los años produce daño tisular permanente y progresivo afecta el aspecto físico, el funcionamiento de los órganos y el desarrollo mental.

Estos pacientes presentan afecciones del sistema respiratorio, cardíaco, deformidades musculoesqueléticas lo que representa un verdadero desafío anestesiológico.

Caso clínico: Paciente sexo femenino de 30 años consulta por tumoración en zona umbilical de 2 años, en las últimas $48 \mathrm{~h}$ evoluciona con dolor, el equipo quirúrgico decide programar cirugía para hernioplastia con colocación de malla.

Paciente con diagnóstico reciente de MPS tipo I-Scheie sin tratamiento.

Valvulopatías: estenosis aórtica moderada más insuficiencia moderada e insuficiencia mitral moderada.

Examen físico: altura 1,42 cm; peso $90 \mathrm{~kg}$ IMC 44.

Apertura bucal: 2,4 cm macroglosia, circunferencia de cuello $44 \mathrm{~cm}$ Distancia TM 1,6 cm Distancia EM 3,4 CM Mallampati G IV Rigidez cervical con limitación importante de la flexo-extensión.

Ap respiratorio: sat $\mathrm{O} 2$ 96\%.

Ap Cardiovascular: TA160/90 Fc 80 soplo sistólico en foco aórtico, disnea clase funcional III.

Abd Globuloso visceromegalias, hernia umbilical.

Ap Locomotor; miembros sup con abducción limitada, manos en garra rígida.

Columna: Rigidez cervical, Hiperlordosis, escoliosis y cifosis.

Preoperatorio: ta $147 / 95$ fc 88 sat $98 \%$ con cánula a $31 / \mathrm{min}$.

Se realiza anestesia regional; bloqueo neuroaxial subaracnoideo abordaje medial con aguja punta lápiz $n^{\circ} 25 \mathrm{~g}$ entre L4 y L5 con bupivacaína isobárica $0,5 \% 8 \mathrm{mg}$ más fentanilo 20 gamas punción sin complicaciones; cambios hemodinámicos; descenso de la ta 110/87 mmHg fc 120; luego 107/78 mmHg fc 75 durante todo el acto quirúrgico (90 min). Paciente egresa a sala común. No se verificaron complicaciones hemodinámicas ni nerviosas, evolución favorable, alta médica a las 36 horas.

Discusión: En las MPS se requieren intervenciones quirúrgicas y el anestesiólogo puede encontrarse con problemas en el manejo de la vía aérea con alta mortalidad, necesidad de re intubación y traqueotomía de urgencia. Actualmente hay escasa información que demuestre la superioridad de una técnica anestésica sobre otra.

Conclusión: En este caso realizamos anestesia regional con bupivacaína isobárica al 0,5\% a dosis bajas considerando sus valvulopatías y la anatomía del paciente, ante la posibilidad de fracaso de la técnica pensamos en anestesia general abordando la vía aérea con fibroscopía o acceso quirúrgico de emergencia. Recomendamos una exhaustiva valoración pre anestésica y manejo anestésico individualizado.

https://doi.org/10.25237/congresoclasa2019.39 